\title{
In Vitro Dissolution Studies on Naproxen-PVP Nanoformulations Show Enhanced Oral Bioavailability of Naproxen
}

\author{
Sandeep Patnaik ${ }^{1}$, Aditya D Kurdekar ${ }^{2}$, LA Avinash Chunduri ${ }^{1}$, C Prathibha ${ }^{2}$ and K Venkataramaniah ${ }^{2 *}$
}

${ }^{1}$ Andhra Pradesh Medtech Zone, India

${ }^{2}$ Laboratories for Nanoscience and Nanotechnology Research, Sri Sathya Sai Institute of Higher Learning, India

*Corresponding author: Kamisetti Venkataramaniah, Laboratories for Nanoscience and Nanotechnology Research, Sri Sathya Sai Institute of Higher Learning, Prasanthinilayam 515134, Andhra Pradesh, India, Tel: +91-9490474571

\begin{abstract}
Phase solubility behavior, physico-chemical characteristics, cytotoxicity, morphology and dissolution enhancement of Naproxen-PVP Nanoformulations have been studied. Drug-polymer ratios of 1:1, 1:2, 1:3, 1:4 for the poorly water-soluble drug, naproxen and the polymer PVP are prepared via wet milling using a conventional Retsch Planetary ball mill. The highest dissolution enhancement over that of the pure drug, $310 \%$ was achieved for the formulation ratio of $1: 4$. This ability of hydrophillic surfactant carriers to accelerate the in vitro dissolution of poorly water-soluble drugs has been attributed to wetting, solubilization and/or deflocculation. The Korsmeyer-Peppas model most aptly fits the in vitro dissolution data indicating a possible drug release mechanism predominated by anomalous non-Fickian diffusion thus helping improve the physicochemical characteristics of naproxen towards its dissolution enhancement and possibly increase the oral bioavailability of the drug without any adverse cytotoxic consequences.
\end{abstract}

\section{Keywords}

Naproxen, PVP, Nanoformulations, Phase-stability, Dissolution, Bioavailability

\section{Introduction}

Naproxen is one of the most popular Non-Steroidal Anti-inflammatory Drugs (NSAIDs), widely administered against a wide range of inflammatory and analgesic disease conditions such as fever, inflammation, and pain related to a variety of muscular and skeletal disorders including osteoarthritis, bursitis, rheumatoid arthritis, kidney stones, ankylosing spondylitis, psoriatic arthritis, gout, menstrual cramps, tendinitis, and migraine. It was first synthesized from the starting material 2-me- thoxynaphthalene (nerolin) [1]. Currently, Naproxen is predicted to be one of the fastest-growing APIs among the adult systemic analgesics and is expected to reach nearly US\$1 billion in retail value sales by 2018 [2].

Chemically speaking, Naproxen is a propionic acid derivative. It is a member of the profen (2-arylpropionic acid). Chemically it is also called (+)-(S)-6-Methoxy-alpha-methyl-2-naphthaleneacetic acid or S)-(+)-2-(6-Methoxy-2-naphthyl) propionic acid. The API is pale white in color and is an odorless, crystalline substance and is highly lipophilic and practically insoluble in aqueous media. The drug, when orally administered, has quite some undesirable side effects like hemorrhage and ulceration of the stomach. As a consequence of its scarce wettability and very poor water-solubility $(0.025$ $\mathrm{mg} / \mathrm{ml}$ at $25^{\circ} \mathrm{C}$ ), it exhibits low and/or variable bioavailability after oral administration. Several approaches were tried to adequately improve the naproxen dissolution properties, and bioavailability. An improved naproxen formulation with quick drug release pattern would be highly useful in the treatment of inflammatory and painful states of the body, like rheumatoid arthritis.

Solid dispersions with polyethylene glycol [3] or polyvinylpyrrolidone [4] or complexation with cyclodextrins $[5,6]$ and liquisolid technique [7] have been reported. In fact, the first bi-component formulations of the drug involved complexation with 2-hydroxylpropyl- $\beta$-cyclodextrin. Lee, et al. [8] have reported these complexes to have increased dissolution characteristics as well as decreased gastrointestinal toxicity when administered orally. Several polymers including HPMC and PVP have been used to

Citation: Patnaik S, Kurdekar AD, Chunduri LAA, Prathibha C, Venkataramaniah K (2018) In Vitro Dissolution Studies on Naproxen-PVP Nanoformulations Show Enhanced Oral Bioavailability of Naproxen. Int J Med Nano Res 5:023. doi.org/10.23937/2378-3664/1410023

Accepted: October 06, 2018: Published: October 08, 2018

Copyright: (c) 2018 Patnaik S, et al. This is an open-access article distributed under the terms of the Creative Commons Attribution License, which permits unrestricted use, distribution, and reproduction in any medium, provided the original author and source are credited. 
dose naproxen were employed to improve the dissolution characteristics of naproxen. Binary co-ground mixtures with drugs like cimetidine [9] and ibuprofen [10] have also been explored for improved solubility of naproxen.

Formulation of naproxen-PLGA nanoparticles by using the single emulsion-solvent evaporation/extraction process have been reported by Javadzadeh, et al. [11]. They were able to improve the physicochemical characteristics of the drug and an increase in the anti-inflammatory effects of the drug following its ocular or intra-joint administration. Liversidge, et al. [12] by reducing drug particle size to $270 \mathrm{~nm}$ and stabilizing the particles in suspension with pluronic F-68 demonstrated the reduction in gastric irritation while the rate of $a b$ sorption increased. The increase in the rate of absorption was attributed to an increase in surface area for dissolution for the Nano Crystal formulation.

Larger the surface area, higher will be the dissolution rate. Since the surface area increases with decreasing particle size, which can be accomplished by conventional methods like trituration, grinding, ball milling, fluid energy micronization, salt formation and controlled precipitation. Although these conventional methods have been used commonly to increase dissolution rate of drug, there are practical limitation with these techniques as the desired bioavailability enhancement may not always be achieved. Therefore, formulation approaches are being explored to enhance bioavailability of poorly soluble drugs. Some such formulation approaches that have been shown to significantly enhance absorption of such drugs is to formulate nanosuspensions.

Nanosuspensions have been reported to be advantageous because of easy industrial scalability, economic viability, high drug loading efficiency, and low excipient side effects $[13,14]$. A simple top down approach explored for drug-nanoformulations (NFs) is the use of a planetary ball mill to break the drug crystals into smaller drug particles [15-17]. The frictional forces and the impact forces caused by the planetary rotations and revolutions of the milling jar containing the drug suspension are responsible for mechanical attrition of the contents of the milling jars [16]. In this work, we explore the use of this very simple and easily scalable wet ball milling technology to design novel NFs of the poorly soluble drug naproxen with the polymer PVP in terms of phase solubility analysis to detect purity of substance, chemical structure by FTIR analysis, structure analysis by PXRD, DSC and morphology by FESEM, in vitro cell viability studies for cytotoxicity and dissolution measurements for the best drug-polymer ratio.

PVP, also called povidone or kollidon ${ }^{\circledR}$, is available in variable molecular weights (determined by their $k$ values), ranging from K-12 to K-90. PVP has been widely explored as carrier for drug molecules [2,10-16]. One of the earliest and easiest means of enhancing the bioavailability of a drug is to improve its dissolution by dispers- ing them in matrices of soluble Kollidon ${ }^{\circledR}$ grades. These polymers form water-soluble complexes with many drug molecules, depending on the chemical structure of the APIs. The exceptional aqueous solubility of the Kollidon ${ }^{\circledR}$ grades along with their ability to form water-soluble complexes with the APIs are often exploited for enhancement of the dissolution characteristics of drugs as they possess admirable hydrophilization abilities, are available in variable molecular weights (determinable by their $\mathrm{k}$ values) for different viscosities, form water-soluble complexes with many APIs, in contrast to most other carrier materials, and are almost universally soluble.

\section{Materials and Methods}

\section{Materials}

Naproxen was obtained as gift sample from SKN Organics, Chennai and Polyvinylpyrrolidone (PVP) Kollidon (K 25) was obtained from BASF, Mumbai. All other chemicals used were of analytical grade and used without further purification.

\section{Synthesis of naproxen Nanoformulations (NFs)}

The NFs were prepared via wet milling using a conventional Retsch Planetary ball mill in various ratios of drug to polymer $(1: 1,1: 2,1: 3,1: 4)$ [18].

The drug and polymer (in the required ratios) were introduced into an agate milling chamber containing $1 \mathrm{~mm}$ agate balls. $40 \mathrm{~mL}$ of $0.5 \%$ aqueous solution of Tween 80 (surfactant) was added to fill the chamber. The samples were co-milled at 500 rpm for 6 hours. Regular breaks of 5 minutes were provided after every 15 minutes of milling to avoid overheating caused due to the high energy involved in the milling process. The high shear force generated by the collision of the agate balls with the solid drug particles fractures the drug crystals into smaller particles and thus nanosuspensions were formed [17]. Tween 80, also called Polysorbate 80 , is a nonionic surfactant with hydrophilic properties incorporated in the formulations to prevent aggregation and provide stability.

The nanosuspensions thus formed by co-milling were lyophilized for about 24 hours and gently powdered manually using a mortar and pestle to obtain free flowing powders. To protect the nanoparticles from damage, due to ice formation and to minimize the particle size growth during lyophilization, mannitol ( $0.1 \%$ by weight) was added as a cryoprotectant prior to lyophilization.

The several formulations of naproxen designed and studied are listed in Table 1.

Table 1: Different nanoformulations of naproxen-PVP.

\begin{tabular}{|l|l|l|l|}
\hline S. No & $\begin{array}{l}\text { Formulation } \\
\text { Label }\end{array}$ & $\begin{array}{l}\text { Drug- carrier } \\
\text { ratio }\end{array}$ & $\begin{array}{l}\text { Percentage of } \\
\text { carrier }\end{array}$ \\
\hline 1 & NP 1 & $1: 1$ & $50 \%$ \\
\hline 2 & NP 2 & $1: 2$ & $66 \%$ \\
\hline 3 & NP 3 & $1: 3$ & $75 \%$ \\
\hline 4 & NP 4 & $1: 4$ & $80 \%$ \\
\hline
\end{tabular}




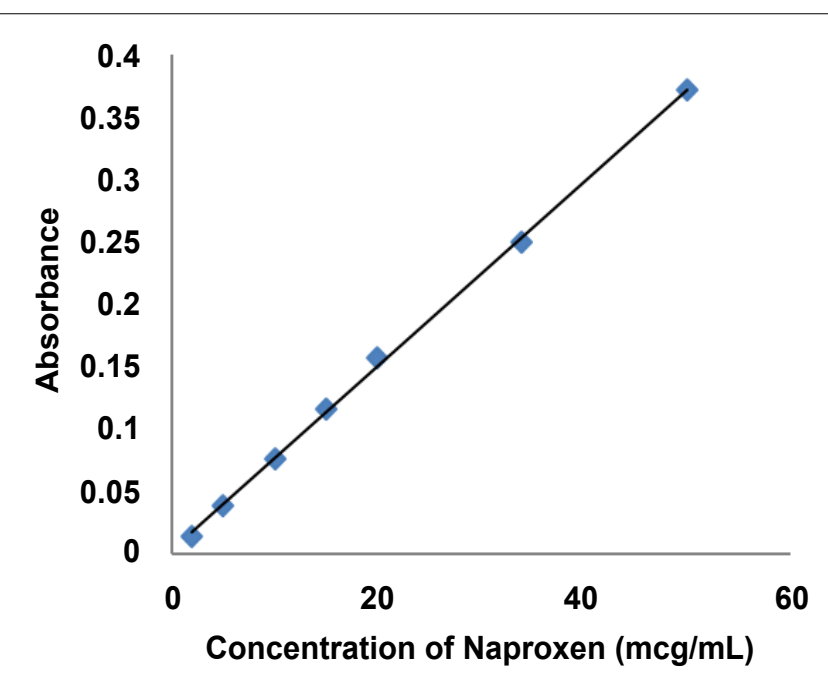

Figure 1: Calibration curve used for spectrophotometric determination of naproxen using Beer Lambert's Law.

\section{Phase solubility studies}

Phase solubility profiles of naproxen in various concentrations of the polymer were established by the method established by Higuchi and Connors [19]. The procedure used was as follows:

To Erlenmeyer flasks $(250 \mathrm{~mL}$ ) containing $25 \mathrm{~mL}$ of the various polymer solution $(0.1 \%, 0.25 \%, 0.5 \%, 0.75 \%$ and $1 \%, w / v)$, an excess amount of drug ( $1 \mathrm{~g})$ was added. The flasks were suitably sealed and shaken at 100 rpm in orbital shaker-incubator for 48 hours at $37^{\circ} \mathrm{C}$. They were left in the incubator for another 24 hours for equilibrium to be established. $5 \mathrm{ml}$ of the supernatant solution was withdrawn and filtered. The amount of drug in the filtrate was photometrically analyzed spectrophotometrically at $278 \mathrm{~nm}$ for determination of the naproxen content using the calibration curve illustrated in Figure 1. The studies were carried out 5 times.

Gibbs free energy of transfer $\left(\triangle G_{t r}^{0}\right)$ values indicate whether the particular treatment is favorable for the solubilization of the drug in an aqueous medium [20]. The more negative the value, the more the spontaneity of the solubilization process. The $\triangle G_{t r}^{0}$ values of Naproxen were computed from the data obtained from phase solubility studies using the following equation [20,21]:

$$
\triangle G_{t r}^{0}=-2.303 R T \log \frac{S_{0}}{S_{s}}
$$

where;

$\mathrm{S}_{\mathrm{o}}=$ molar solubility of naproxen in distilled water

$\mathrm{S}_{\mathrm{s}}=$ molar solubility of naproxen in the presence of PVP 30K

$$
\begin{aligned}
\mathrm{R} & =8.31 \mathrm{JK}^{-1} \mathrm{~mol}^{-1} \\
\mathrm{~T} & =\text { temperature in degree kelvin }
\end{aligned}
$$

\section{Physico-chemical characterizations}

Fourier transform infrared (IR) spectroscopy: FTIR spectra were obtained using an FTIR Spectrophotometer (Spectrum FTIR (Scimadzu, IRAffinity-1)). The spectrum was recorded in the range of $4000-400 \mathrm{~cm}^{-1}$. The procedure consisted of dispersing a sample in $\mathrm{KBr}$ followed by gentle mixing. The spectrum was scanned at a resolution of $0.15 \mathrm{~cm}^{-1}$ and scan speed was $20 \mathrm{scan} / \mathrm{s}$.

Differential Scanning Calorimetry (DSC): Differential Scanning Calorimeter (TGA DSC 1, Mettler Toledo) was used to characterize the thermal behavior of the samples. The samples were heated at a rate of $10^{\circ} \mathrm{C} \mathrm{min}^{-1}$ from ambient temperature to the melting point.

X-ray Diffraction (XRD): XRD patterns were recorded using PANalytical X'pert Pro MPD diffractometer, with the following settings: $\mathrm{Cu} K \alpha$ radiation with wavelength $1.54 \hat{A}$, voltage $=45 \mathrm{kV}$, current $=40 \mathrm{~mA}$. Measurements were made in the $2 \theta$ range of 10 to $80^{\circ}$.

Field Emission Scanning Electron Microscopy (FESEM): Double sided carbon tapes were fixed on an aluminum stub. The powders were sprinkled onto the tape and gold sputtered for 10 mins. The aluminum stub was placed in the vacuum chamber of a scanning electron microscope (Carl Zeiss SMT- Super Ultra Model Gemini Ultra 55) operated at $4 \mathrm{kV}$. The particles were observed for surface characteristics.

\section{In vitro cytotoxicity studies}

Caco 2 cell culture: The cell culture experiments were performed in a biosafety level II cabinet hood. The Caco-2 cells of passage number $12-25$ were cultured using minimum essential medium, MEM (pH 7.4) in culture plates at $37^{\circ} \mathrm{C}$ in a $\mathrm{CO} 2$ incubator. The other supplements added to the medium were heat inactivated fetal bovine serum, sodium bicarbonate, antimycotic and antibacterial mixture and gentamycin. The medium was changed every alternate day till the cells became $80 \%$ confluent. The washing of the cells were done using phosphate buffer saline and the removal of cells were performed by trypsinization ( $0.25 \%$ trypsin, $0.2 \%$ EDTA solution) at $37^{\circ} \mathrm{C}$ for $7-10$ mins. The cells obtained from trypsinization were obtained as a pellet by centrifugation for 3 minutes at $200 \times \mathrm{g}$ and resuspended into the MEM and used for sub-culturing or cytotoxicity studies.

Cytotoxicity studies: The resuspended cells obtained after trypsin treatment of an $80 \%$ confluent plate were seeded at a cell density of $4 \times 103$ in a 96 well plate for 48 hours and successively exposed to different concentrations of the carriers and the NFs made by the culture medium. Culture medium was changed after 48 hours of incubation and the test solutions were added. The cytotoxicity was assessed from the cell viability data obtained from the MTT [(3-(4, 5-dimethylthiazol-2-yl)-2,5-diphenyltetrazolium bromide] cell proliferation assay.

After 48 hours of incubation, $20 \mu \mathrm{L}$ of the MTT reagent was added to each well. The plates were then 
incubated for another 4 hours. Then the medium was removed and the intracellular formazan was solubilized with $100 \mathrm{~mL}$ of dimethyl sulfoxide (DMSO). The absorbance was recorded using a microplate reader (Spectramax M5) at $490 \mathrm{~nm}$. Cell viability was assessed as the percentage of absorbance of the analyte with respect to the control. The statistical significance of the difference with respect to control was investigated by the twotailed Student's t-test.

\section{In vitro dissolution testing}

The in vitro dissolution analyses were performed using a USP type II dissolution testing paddle apparatus (DBK Dissolution Tester, Mumbai, India). A known amount of sample (equivalent to $50 \mathrm{mg}$ of naproxen) was introduced into the glass jar of the USP type II paddle apparatus containing $900 \mathrm{~mL}$ of simulated intestinal fluid (phosphate buffer, $6.8 \mathrm{pH}$ ). This was stirred at $70 \mathrm{rpm}$ for 2 hours. After predetermined regular intervals, $3 \mathrm{~mL}$ aliquots of the sample were withdrawn, filtered, suitably diluted and the concentrations of the withdrawn solutions were determined using a UV spectrophotometer (Schimadzu, UV 2450). To maintain a constant volume during dissolution, $3 \mathrm{~mL}$ of solution was replaced into the glass jar after every withdrawal. Corrections for this dilution were made during the calculations. The percentage of the drug dissolved was calculated and plotted versus time. These studies were carried out three times.

\section{Results and Discussion}

\section{Phase solubility studies}

Figure 1 shows the influence of increasing concentration of the carriers on the solubility of naproxen in simulated gastric fluid ( $\mathrm{pH} 1.2)$ and simulated intestinal fluid ( $\mathrm{pH}$ 6.8). Pure Naproxen exhibited higher solubility in SIF $(49.12 \mu \mathrm{g} / \mathrm{mL})$ than in SGF $(2.42 \mu \mathrm{g} / \mathrm{mL})$. This could be attributed to the fact that naproxen is a weak acid with a $\mathrm{pK}_{\mathrm{a}}$ value of 4.15 . So, percentage of naproxen ionized would be much more in SIF than in SGF. The drug, thus, exhibits $\mathrm{pH}$ dependent solubility [7]. The phase solubility data show a linearly increasing trend in naproxen solubility with increasing carrier levels (Figure 1). The high solubilizing effect of PVP could be attributed to the multiple interaction sites in its chain and its hydrophilic property [22]. The solubility of a drug in dissolution media can be influenced by altering different physicochemical properties, like hydrophobicity/hydrophilicity, viscosity, chemical structure and polarity, etc.

The incorporation of PVP in drug formulations has been reported to increase the dissolution rate of poorly soluble drugs such as aceclofenac [17], piroxicam [23], and glibenclamide [24] leading to an improved drug bioavailability. The $\triangle G_{t r}^{0}$ values obtained from the phase solubility curves with several carriers are listed in Table 2 . The most negative value of $\triangle G_{t r}^{0}$ is obtained for the solution with PVP which is indicative of fact that the process of transfer of naproxen from the bulk medium to its aqueous solutions was most favorable.

\section{Characterization}

FTIR analysis: FTIR spectra of pure naproxen, all the NFs and the carriers were recorded using an FTIR Spectrophotometer (Spectrum FTIR (Scimadzu, IRAffinity-1)). in the range of $4000-400 \mathrm{~cm}^{-1}$. The sample was in $\mathrm{KBr}$ followed by gentle mixing. The spectrum was scanned at a resolution of $0.15 \mathrm{~cm}^{-1}$ and scan speed was 20 scans per second.

As evident from the Figure 2, the polymer PVP showed a vibrational band at $1658 \mathrm{~cm}^{-1}$ attributable to the $\mathrm{C}=\mathrm{O}$ stretching of its amide group. The NFs show that with increase in polymer content there was a decrease in the intensity of the $\mathrm{C}=\mathrm{O}$ stretch (monomer) of naproxen and complete disappearance of the dimeric stretch peak. The NFs with lower PVP content an additional doublet at $1630 \mathrm{~cm}^{-1}$ corresponding to the $\mathrm{C}=\mathrm{O}$ stretch of PVP hydrogen bonded to the carboxylic acid-OH group of NAP. The NF with higher PVP content showed a singlet peak at $1673 \mathrm{~cm}^{-1}$. This indicates that the drug particles are predominantly stabilized in the PVP matrix by $\mathrm{H}$-bonding.

PXRD analysis: As can be seen in Figure 3, distinct sharp peaks of naproxen were obtained at the diffraction angles $14.50,17.73$ and 27.45 . Thus, the crystalline nature of pure naproxen is evident from its PXRD spectrum.

Table 2: $\Delta \mathrm{G}_{\mathrm{tr}}^{0}$ (joules $/ \mathrm{mol}$ ) obtained from the phase solubility studies for the carrier PVP.

Percentage of $\Delta \mathbf{G}^{0}{ }_{\text {tr }}$ (joules $/ \mathrm{mol}$ ) for $\Delta \mathbf{G}^{0}{ }_{\text {tr }}$ (joules $/ \mathrm{mol}$ ) for Polymer (W/V) the different carriers the different carriers

\begin{tabular}{|l|l|l|}
\hline & at $37^{\circ} \mathbf{C}$ in SGF. & at $37^{\circ} \mathbf{C}$ in SIF \\
\hline 0.1 & -2186.471 & -350.717 \\
\hline 0.25 & -4539.736 & -640.407 \\
\hline 0.5 & -5564.670 & -1093.34 \\
\hline 0.75 & -6191.089 & -1366.64 \\
\hline 1.0 & -6687.629 & -1624.05 \\
\hline
\end{tabular}

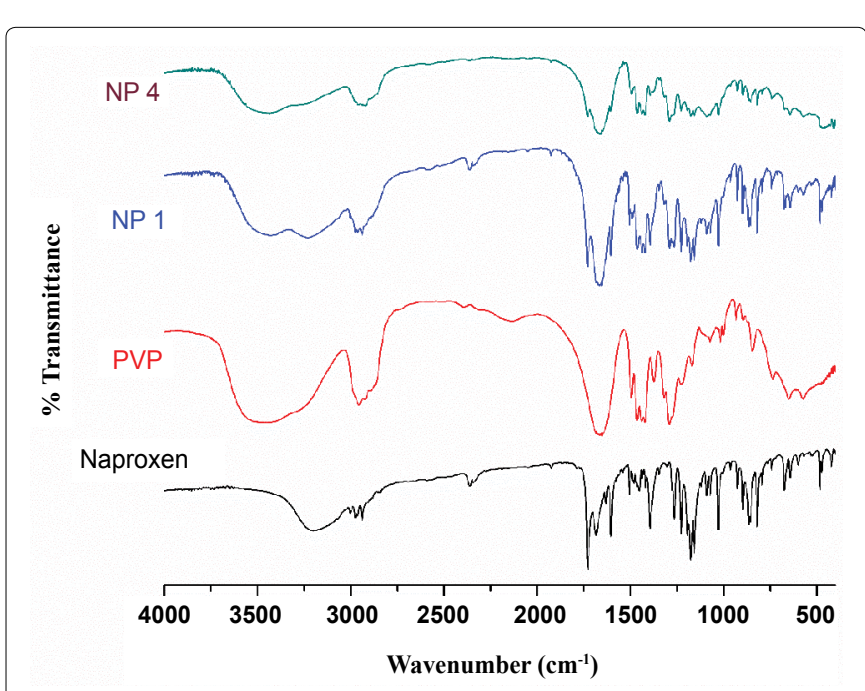

Figure 2: FTIR spectra of naproxen, PVP K 25 and the nanoformulations. 


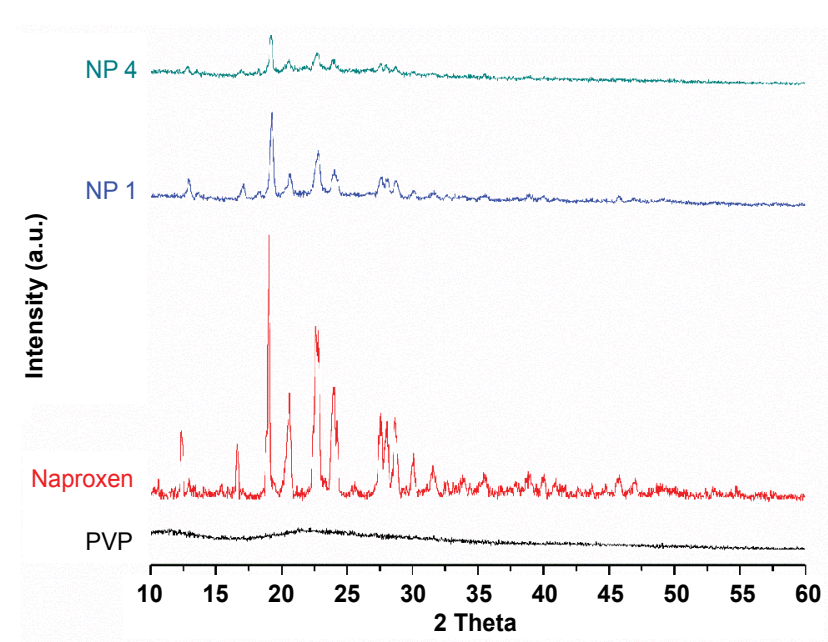

Figure 3: XRD spectra of naproxen, PVP K25 and the nanoformulations.

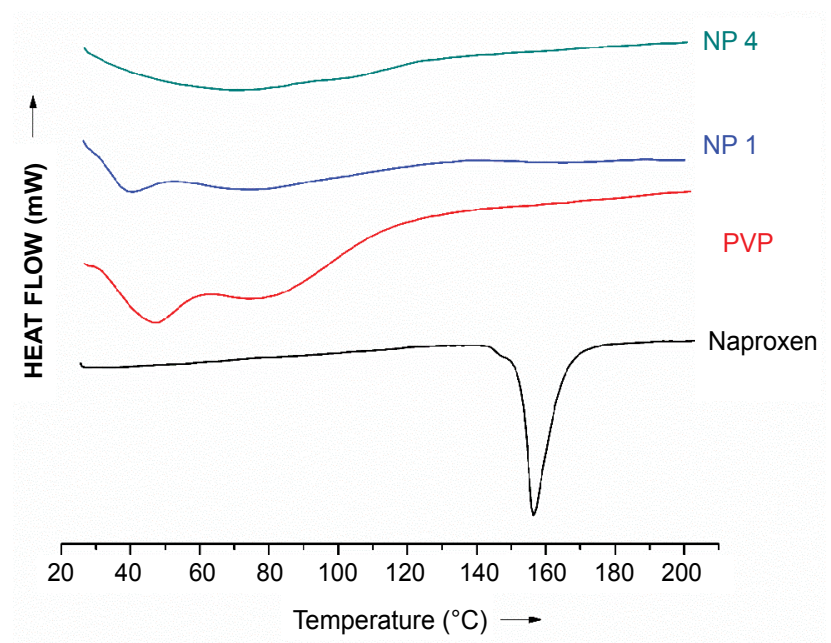

Figure 4: DSC curves of naproxen, PVP and the nanoformulations.
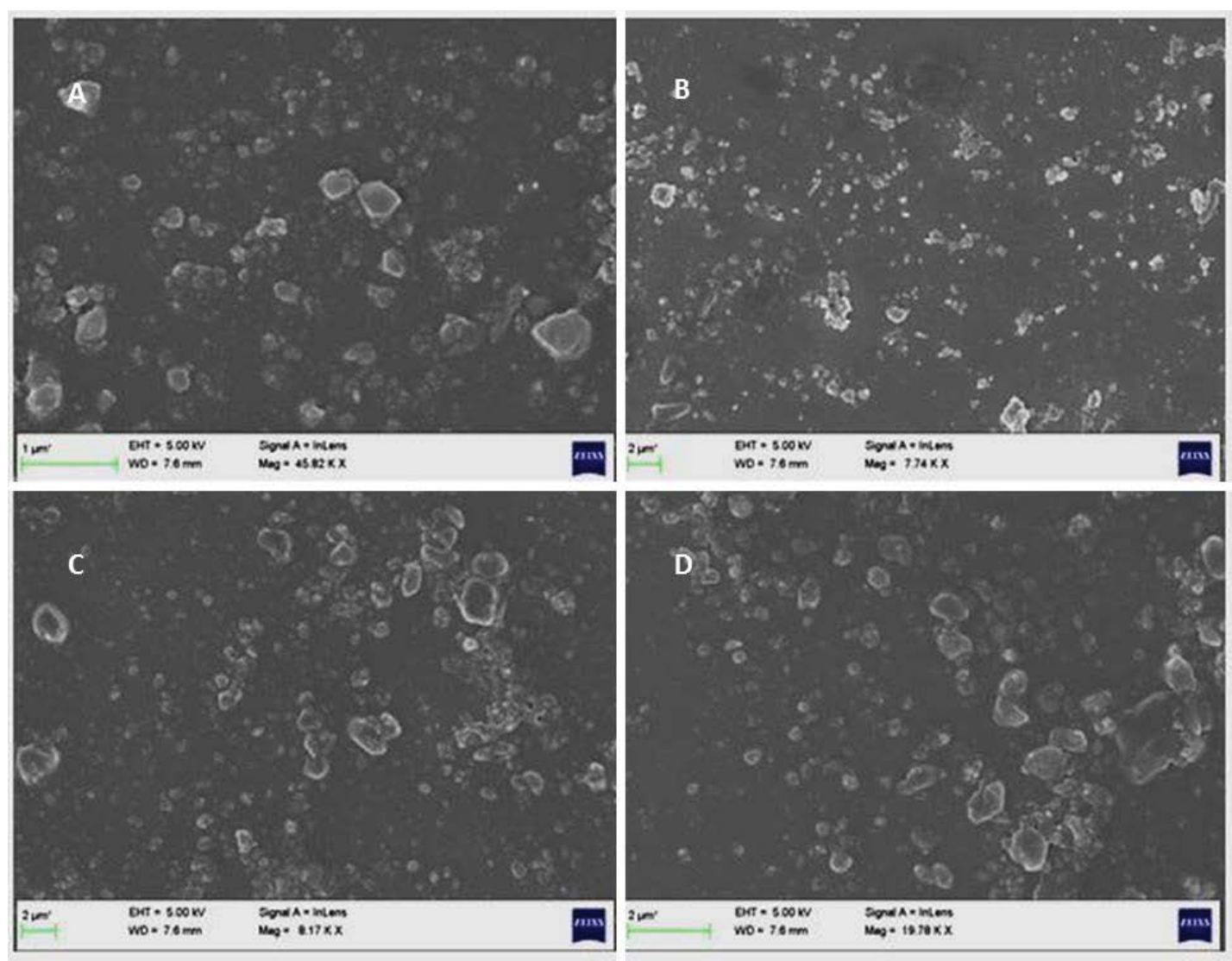

Figure 5: FESEM images of naproxen: PVP nanoformulations NP 1 ( $A$ and $B$ ) and NP 4 (C and D).

The spectrum of PVP shows two broadened halos at $2 \theta$ values of 12 and 22 . The positions of the diffraction peaks of naproxen in all the nanosuspensions were almost completely superimposable, indicating that there was no formation of any other crystal morphology during the milling process. However the lowered intensity and slight broadening of the peaks in the various formulations suggests decreasing crystallite sizes of the drug dispersed in the polymer with increase in the polymer to drug ratio.

DSC analysis: The DSC curve of PVP $\mathrm{K} 25$ (Figure 4) shows a broad endotherm with a peak at $47.3^{\circ} \mathrm{C}$ (due to the loss of water from its hygroscopic polymeric chains) and a glass transition peak $\left(\mathrm{T}_{\mathrm{g}}\right)$ at $88^{\circ} \mathrm{C}$. The TA curves of the NFs showed lowered $T_{g}$ of the polymer and almost negligible onset melting point of the drug. Though the melting endotherm is undetectable in the TA curve, XRD of the NFs of PVP show the prevalence of crystallinity in the systems. This apparent incoherence could arise from the fact that PVP has a low $T_{g}$ value which when reached during the thermal program could cause the dissolution of the drug in the molten polymer.

Morphology: Representative FESEM images of the NFs of Naproxen and PVP K 25 obtained from wet ball milling are shown Figure 5 corresponding to low (NP 1) and high polymer content (NP 4) at different magnifications suggest that the amount of carrier had a great 


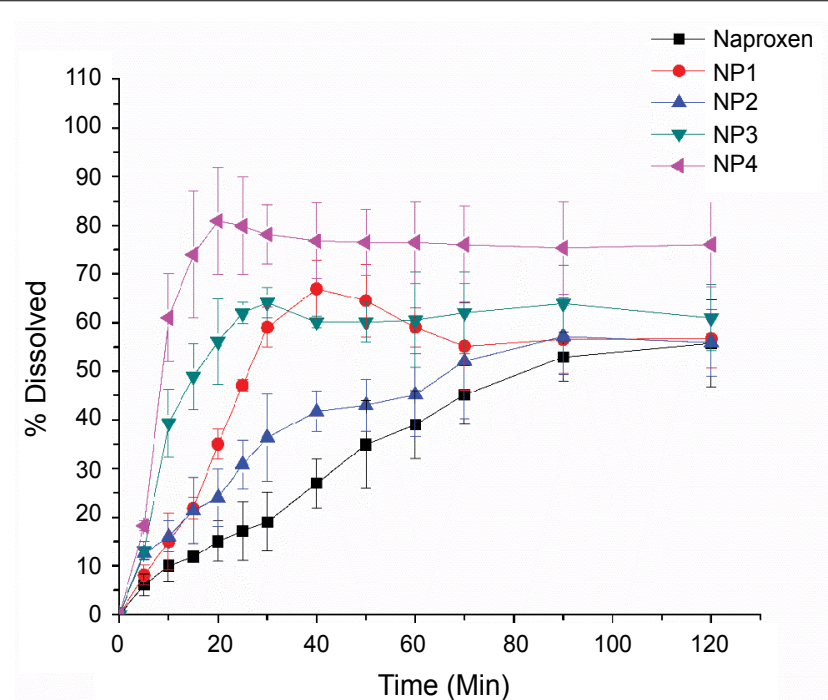

Figure 6: Dissolution proflies of naproxen-PVP nanoformulations.

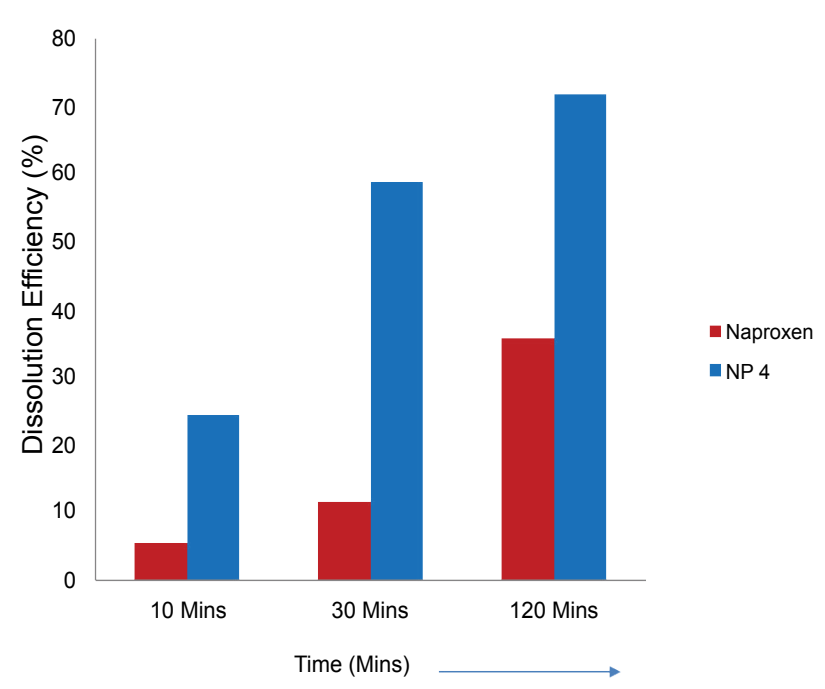

Figure 7: Dissolution efficiencies calculated at different time for the various nanoformulations of naproxen-PVP.

Table 3: Percentage dissolution efficiency.

\begin{tabular}{|c|c|c|c|c|c|c|}
\hline \multirow[t]{2}{*}{ Formulation } & \multicolumn{6}{|c|}{$\%$ Dissolution Efficiency } \\
\hline & $5 \mathrm{~min}$ & $10 \mathrm{~min}$ & $20 \mathrm{~min}$ & $30 \mathrm{~min}$ & $90 \mathrm{~min}$ & $120 \mathrm{~min}$ \\
\hline Pure Naproxen & 3.051 & 5.556 & 8.871 & 11.607 & 29.542 & 35.740 \\
\hline NP 1 & 6.702 & 18.010 & 37.175 & 48.711 & 65.928 & 68.316 \\
\hline NP 2 & 7.003 & 19.910 & 42.102 & 51.001 & 67.234 & 70.206 \\
\hline NP 3 & 8.365 & 22.092 & 45.776 & 54.234 & 69.023 & 69.546 \\
\hline NP 4 & 9.097 & 24.350 & 48.419 & 58.852 & 70.515 & 71.808 \\
\hline
\end{tabular}

influence on the morphology of the NFs. The particles were of varied morphology with particle shapes ranging from irregular, cuboidal to round. The size distribution varied from about $50 \mathrm{~nm}$ to $300 \mathrm{~nm}$. Since ball milling is top down synthetic route, it offers poor control over morphology and size distribution. All the formulations showed similar characteristics in terms of morphology and particle size.

\section{Dissolution studies}

As can be seen in Figure 6 and Table 3 at the end of 30 minutes, PVP based NP4 formulation could achieve a $310 \%$ improvement in its dissolution. The dissolution profiles of PVP based NFs show decrease in supersaturation after 30 mins in SIF and SGF indicating the possibility of recrystallization of the drug as can be expected from the XRD studies. The carrier matrix of PVP could not prevent the recrystallization of the drug in spite of $\mathrm{H}$ bonding stabilization. This could be partly due to the hygroscopic nature of PVP. However, increase in PVP content in the formulations could help prevent the recrystallization.

Figure 7 and Table 3 show the \%DE values of the Nanoformulation NP 4 in comparison with that of the pure drug at three different time scales representing early and late phase of dissolution. At $t=10$ early phase of dissolution, NP 4 shows an enhanced D.E value of $30 \%$. This is a 4 -fold enhancement of the efficiency compared to that of pure drug. Similarly, at $\mathrm{t}=30 \mathrm{mins}$, the $\%$ DE increased from $11 \%$ to $70 \%$. An efficiency en- hancement of $310 \%$ was achieved by NP 4 at $t=120$ in comparison with that of the pure drug.

\section{Cytotoxicity studies on naproxen nanoformulations}

Caco-2 cells were used as in vitro models to assess the cell viability characteristics of the carrier PVP and the nano-formulations of Naproxen. The assessment of cell viability was done using the tetrazolium salt based MTT assay. Duration of 12 hour exposure was selected because scintigraphic gastric transit studies in humans suggest they are physiologically relevant average and maximum exposure times, respectively, in the gastrointestinal tract. All the NFs were observed to follow the cytotoxicity trend of the polymers. Our results show that no significant decrease in cell viability was seen until $0.01 \%$ concentration of PVP for 12-h exposure. The NFs as well as the polymer alone had no significant effect on the viability of Caco- 2 cells below $0.01 \%$ concentrations. Here it is important to note that intact intestinal membranes (in vivo) often are found to be more resistant to the cytotoxic effects of excipients than are cell culture models (in vitro). The intestine has a protective mucous layer, whereas the cell culture monolayers do not. The intestinal tissues also have more capacity to recover from trauma than the cultured cells. Hence the present NFs can be expected to show lesser cytotoxicity when subjected to in vivo studies.

The dissolution of naproxen from all the NFs with PVP was significantly faster than that of the pure drug in both the dissolution media. Also, increase in the poly- 
mer content in the formulations had an enhanced effect on the dissolution of naproxen. The release from NFs visually revealed the tendency of the drug to leave the surface of the dissolution medium instantaneously and disperse in the bulk of the medium indicating the occurrence of rapid wetting. There was no visible decline in the supersaturation in case of the NFs with higher PVP content even at the end of two hours. The high solubility enhancement from PVP could be attributed to micellar solubilization and/or reduction of activity coefficient of the drug through reduction of hydrophobic interaction(s).

\section{Dissolution efficiency}

The Noyes-Whitney equation [25] is often used explain the dissolution results.

$$
\frac{d C}{d t}=\operatorname{DS} \frac{\left(C_{s}-C\right)}{h}
$$

where $\mathrm{dC} / \mathrm{dt}$ is the dissolution rate, $\mathrm{D}$ is the diffusion coefficient of the dissolved drug particles, which is a parameter viscosity of the dissolution medium; $\mathrm{S}$ represents the exposed surface area to dissolution; $h$ is the thickness of the diffusion layer, which is a parameter affected by agitation; Cs is the saturation solubility of the drug in solution in the diffusion layer, the term $\mathrm{C}$ is the concentration of the drug in the dissolution medium. Since the dissolution tests were performed under the same stirring conditions (70 rpm) and the dissolution media was prepared with same viscosity, the parameters in the equation $h$ and $D$ can be assumed to be constant. Thus, the only terms affecting the dissolution rates of the Nanoformulations can be assumed to be $\left(C_{s}-C\right)$. It can, therefore, be concluded that the wettability of the drug particles were increased and the particle size decreased. The enhancement in the dissolution of the Nanoformulations could thus be attributed to a combined effect of decrease in size of the drug as well as improved wetting characteristics of the polymer. For a comparative analysis of the drug release from the formulations, \%DE values at several times, representing the various phases of dissolution study, were computed (Table 3).

Dissolution efficiency (DE) is the area under the dissolution curve within a given range of time. Lower \%DE values observed for NS1 could probably be attributed to slow emulsification process leading to slower dissolution in the initial time periods. At the end of $120 \mathrm{~min}$ NS4 achieved an improvement of $167 \%$. Lower \%DE values observed for NS1 could probably be attributed to slow emulsification process leading to slower dissolution in the initial time periods.

\section{Mathematical modeling}

The dissolution profiles from formulations have been treated with several mathematical models to describe release rates and mechanisms, their utility being dependent on the nature of dosage. When drug release rate is proportional to the drug remaining in the dosage form, dissolution can be said to follow first order release kinetics. The data obtained from the dissolution analysis were fitted into various mathematical models listed in Table 4 to describe the drug release mechanism from the different formulations.

The release kinetics of a drug can be influenced by several parameters. For a poorly water-soluble drug, like naproxen, release kinetics should be predominantly guided by erosion of the matrix. The analysis of the data obtained from the dissolution studies with mathematical formulae helps relating the results as a function of the formulation characteristics. The analysis of the data has been done on some empirical drug release equations.

Table 4: Mathematical models for studying the drug release kinetics from dissolution profiles.

\begin{tabular}{|l|l|l|l|}
\hline S.No & Model & Equation & Plot \\
\hline 1. & Zero order & $\operatorname{Ln}\left(\frac{M_{0}}{M_{t}}\right)=\mathrm{K}_{0} t$ & Cumulative amount of drug released versus time. \\
\hline 2. & First order & $M_{0}-\mathrm{M}_{t}=\mathrm{K}_{1} t$ & Log of percentage of drug remaining unreleased versus time. \\
\hline 3. & Higuchi & $M_{t}=\mathrm{K} \sqrt{t}$ & Cumulative percent release versus square root of time. \\
\hline 4. & Hixson-Crowell & $\left(M_{0}\right)^{\frac{1}{3}}-\left(M_{t}\right)^{\frac{1}{3}}=\mathrm{K}_{\frac{1}{3}} t$ & $\begin{array}{l}\text { Cube root of drug percentage remaining in the matrix versus } \\
\text { time. }\end{array}$ \\
\hline 5. & Korsemeyer-Peppas & $\frac{M_{t}}{M_{\infty}}=\mathrm{Kt}^{n}$ & Log of the cumulative percent drug release versus log of time. \\
\hline
\end{tabular}

Table 5: Slopes and $\mathrm{R}^{2}$ values obtained from fitting the experimental in vitro dissolution data into the various release kinetic models.

\begin{tabular}{|c|c|c|c|c|c|c|c|c|c|c|}
\hline \multirow[t]{2}{*}{ Formulation } & \multicolumn{2}{|c|}{ Zero Order } & \multicolumn{2}{|c|}{ First order } & \multicolumn{2}{|c|}{ Higuchi [19] } & \multicolumn{2}{|c|}{ Hixson-Crowell } & \multicolumn{2}{|c|}{ Korsemeyer-Peppas [22] } \\
\hline & Slope & $\mathbf{R}^{2}$ & Slope & $\mathbf{R}^{2}$ & Slope & $\mathbf{R}^{2}$ & Slope & $\mathbf{R}^{2}$ & Slope & $\mathbf{R}^{2}$ \\
\hline NP1 & -0.021 & 0.612 & -0.004 & 0.933 & 8.665 & 0.933 & 0.022 & 0.821 & 0.7182 & 0.923 \\
\hline NP2 & -0.087 & 0.652 & -0.019 & 0.921 & 12.123 & 0.811 & 0.032 & 0.621 & 0.722 & 0.966 \\
\hline NP4 & -0.127 & 0.369 & -0.022 & 0.954 & 17.507 & 0.795 & 0.054 & 0.664 & 0.735 & 0.783 \\
\hline
\end{tabular}


Table 5 lists the slopes and $R^{2}$ values obtained from fitting the experimental in vitro dissolution data into the various release kinetic models. The fittings were carried out for the data obtained till 40 minutes for the quick release formulations NP4. Considering only the data points till super saturation was achieved, the data fit well into the first order and the Korsmeyer-Peppas [22] models. According to the regression values, the drug release data were observed to best fit into kinetic models in the order: Korsmeyer-Peppas $\approx$ First order $>$ Higuchi $>$ Hixon-Crowel > Zero order.

This model describes the release of the drug from polymeric matrices based on the release exponent factor ' $n$ ' which is calculated as the slope when log of the percentage of the drug released is plotted versus the log of time. This $n$ value characterizes the nature of different release mechanisms for Fickian diffusion $(n=0.5)$ or non-Fickian/anomalous release (for $0.5<n<1$ ). Since the values of diffusional exponent ' $n$ ', obtained from the fitting, ranged from 0.545 to 0.966 , the release phenomena may be regarded to follow a non-Fickian model.

\section{Conclusion}

A simple and easily scalable top down approach using of a planetary ball mill to break the drug crystals into smaller drug particles has been explored for to design novel NFs of the poorly water-soluble drug naproxen with the polymer PVP. FESEM analysis showed particles of varied morphology with particle shapes ranging from irregular, cuboidal to round with size distribution varying from $50 \mathrm{~nm}$ to $300 \mathrm{~nm}$ and showing that the amount of carrier having a great influence on the morphology of the NFs. The phase solubility data show a linearly increasing trend in naproxen solubility with increasing carrier levels (Figure 1). The high solubilizing effect of PVP could be attributed to the multiple interaction sites in its chain and its hydrophilic property. The drug exhibited $\mathrm{pH}$ dependent solubility.

Caco- 2 cells were used as in vitro models to assess the cell viability characteristics of the carrier PVP and the nano-formulations of Naproxen. All the NFs were observed to follow the cytotoxicity trend of the polymers. Our results show that no significant decrease in cell viability was seen until $0.01 \%$ concentration of PVP for 12-h exposure. The NFs as well as the polymer alone had no significant effect on the viability of Caco- 2 cells below $0.01 \%$ concentrations.

The dissolution profiles of PVP based NFs show decrease in supersaturation after 30 mins in SIF and SGF indicating the possibility of recrystallization of the drug as can be expected from the XRD studies. The carrier matrix of PVP could not prevent the recrystallization of the drug in spite of $\mathrm{H}$ bonding stabilization. This could be partly due to the hygroscopic nature of PVP. However, increase in PVP content in the formulations could help prevent the recrystallization. The highest dissolution enhancement was achieved for the formulation with PVP with ratio of 1:4. This is a $310 \%$ enhancement when compared to that of the pure drug. The ability of hydrophillic carriers to accelerate in vitro dissolution of poorly water-soluble drugs has been attributed to wetting and/or deflocculation. Thus, the nano-formulations studied can help improve the physicochemical characteristics of Naproxen towards its dissolution enhancement and possibly will increase the oral bioavailability of the drug without any adverse cytotoxic consequences leading to easy industrial scalability, economic viability, high drug loading efficiency, and low excipient side effects.

\section{Conflict of Interest}

The authors declare no conflict of interest.

\section{Acknowledgements}

The authors acknowledge with gratitude the inspiration provided by Bhagawan Sri Sathya Sai Baba, the founder Chancellor of Sri Sathya Sai Institute of Higher Learning for encouraging research on problems of societal interest.

\section{Contributions}

The idea was conceived by KV and SP. Material synthesis and batch experiments and analysis was done by $\mathrm{SP}, \mathrm{CP}, \mathrm{LAA}$ and $\mathrm{ADK}$.

\section{Funding}

No funding was received towards the research from any of National or International funding agencies.

\section{References}

1. Harrington PJ, Lodewijk E (1997) Twenty years of naproxen technology. Org Process Res Dev 1: 72-76.

2. (2015) Global and Chinese Naproxen (CAS22204-53-1) Industry.

3. Mura P, Faucci MT, Manderioli A, Bramanti G, Parrini $P$ (1999) Thermal behavior and dissolution properties of naproxen from binary and ternary solid dispersions. Drug Dev Ind Pharm 25: 257-264.

4. Mura P, Faucci MT, Bettinetti GP (2001) The influence of polyvinylpyrrolidone on naproxen complexation with hydroxypropyl-beta-cyclodextrin. Eur J Pharm Sci 13: 187-194.

5. Mura P, Furlanetto S, Cirri M, Maestrelli F, Corti G, et al. (2005) Interaction of naproxen with ionic cyclodextrins in aqueous solution and in the solid state. J Pharm Biomed Anal 37: 987-994.

6. Mura P, Bettinetti GP, Cirri M, Maestrelli F, Sorrenti M, et al. (2005) Solid-state characterization and dissolution properties of Naproxen-Arginine-Hydroxypropyl-cyclodextrin ternary system. Eur J Pharm Biopharm 59: 99-106.

7. Tiong N, Elkordy AA (2009) Effects of liquisolid formulations on dissolution of naproxen. Eur $\mathrm{J}$ Pharm Biopharm 73: 373-384.

8. Lee BJ, Lee JR (1995) Enhancement of solubility and dissolution rate of poorly water- soluble naproxen by complex- 
ation with 2-hydroxypropyl-beta- cyclodextrin. Arch Pharm Res 18: 22-26.

9. Allesø M, Chieng N, Rehder S, Rantanen J, Rades T, et al (2009) Enhanced dissolution rate and synchronized release of drugs in binary systems through formulation: Amorphous naproxen-cimetidine mixtures prepared by mechanical activation. J Control Release 136: 45-53.

10. Bogdanova S, Pajeva I, Nikolova P, Tsakovska I, Müller B (2005) Interactions of poly(vinylpyrrolidone) with ibuprofen and naproxen: Experimental and modeling studies. Pharm Res 22: 806-815.

11. Javadzadeh $Y$, Ahadi F, Davaran S, Mohammadi G, Sabzevari A, et al. (2010) Preparation and physicochemical characterization of naproxen-PLGA nanoparticles. Colloids Surf B Biointerfaces 81: 498-502.

12. Liversidge GG, Conzentino $P$ (1995) Drug particle size reduction for decreasing gastric irritancy and enhancing absorption of naproxen in rats. Int J Pharm 125: 309-313.

13. Müller RH, Jacobs C, Kayser O (2001) Nanosuspensions as particulate drug formulations in therapy: Rationale for development and what we can expect for the future. Adv Drug Deliv Rev 47: 3-19.

14. Patravale VB, Date AA, Kulkarni RM (2004) Nanosuspensions: A promising drug delivery strategy. J Pharm Pharmacol 56: 827-840.

15. Bhakay A, Merwade M, Bilgili E, Dave RN (2011) Novel aspects of wet milling for the production of microsuspensions and nanosuspensions of poorly water-soluble drugs. Drug Dev Ind Pharm 37: 963-976.

16. George M, Ghosh I (2013) Identifying the correlation between drug/stabilizer properties and critical quality attri- butes (CQAs) of nanosuspension formulation prepared by wet media milling technology. Eur J Pharm Sci 48: 142-152.

17. Ghosh I, Michniak-Kohn B (2012) Design and characterization of submicron formulation for a poorly soluble drug: The effect of Vitamin E TPGS and other solubilizers on skin permeability enhancement. Int J Pharm 434: 90-98.

18. Florez-Zamora MI, Martinez-perez CA, Garcia-Guaderrama M, Estrada-Guel I, Espinosa-magana F, et al. (2008) Comparative study of Al-Ni-Mo alloys obtained by mechanical alloying in different ball mills. Rev Adv Mater Sci 18: 301-304.

19. Higuchi T, Connors KA (1965) Phase-Solubility Techniques. Adv Anal Chem Instrum 4: 117-210.

20. Furqan A Maulvi, Sonali J Dalwadi, Vaishali T Thakkar, Tejal G Soni, Mukesh C Gohel, et al. (2011) Improvement of dissolution rate of aceclofenac by solid dispersion technique. Powder Technology 207: 47-54.

21. Ahuja N, Katare OP, Singh B (2007) Studies on dissolution enhancement and mathematical modeling of drug release of a poorly water-soluble drug using water-soluble carriers. Eur J Pharm Biopharm 65: 26-38.

22. Korsmeyer RW, Gurny R, Doelker E, Buri P, Peppas NA (1983) Mechanisms of solute release from porous hydrophilic polymers. Int J Pharm 15: 25-35.

23. Reintjes T (2011) Solubility enhancement with BASF pharma polymers solubilizer compendium.

24. Patnaik S, Aditha SK, Rattan T, Kamisetti V (2015) Aceclofenac-Soluplus nanocomposites for increased bioavailability. Soft Nanosci Lett 5: 13-20.

25. Noyes AA, Whitney WR (1897) The rate of solution of solid substances in their own solutions. J Am Chem Soc 19: 930-934. 